\title{
Characterization of the Relationships between Wheat Cultivars, Fusarium Head Blight, and Mycoflora Grains
}

\author{
Wojciech Pusz ${ }^{12 *}$, Fabio Mascher ${ }^{2}$, \\ Elżbieta Czembor ${ }^{3}$, Jerzy Czembor ${ }^{3,4}$, Rafal Ogórek ${ }^{5}$ \\ ${ }^{1}$ Department of Plant Protection, Wrocław University of Environmental and \\ Life Sciences Wrocław, Poland \\ ${ }^{2}$ Agroscope Institute of Plant Production Sciences (IPS) \\ Nyon, Switzerland \\ ${ }^{3}$ Departament of Grasses, Legumes and Energy Plants, Plant Breeding and Acclimatization Institute - NRI, Poland \\ ${ }^{4}$ National Centre for Plant Genetic Resources, Plant Breeding and Acclimatization Institute - NRI, Poland \\ ${ }^{5}$ Department of Genetics, Institute of Genetics and Microbiology, University of Wrocław, Poland
}

Received: 29 December 2015

Accepted: 20 February 2016

\begin{abstract}
Stored wheat grains are colonized by a wide range of saprophytic and pathogenic fungi. Contamination by mycotoxigenic species like Fusarium spp. raise concern about the food safety of the commodity. Different environmental and genetic factors govern colonization. The present work studies the relationships between the wheat variety and the resistance against Fusarium head blight (FHB) among the diversity of grain colonizers. Five Swiss and five Polish varieties were planted in an experimental site, and the grains were harvested and stored according to a unified protocol in order to avoid contamination during and after harvest. In parallel, the resistance of the varieties was established by conducting trials at two other experimental sites with artificial infections. Overall, about 18 fungal taxa were identified on the grains from the plot trial. Results show significant differences in the presence and abundance of fungal species between the varieties. Colonization, in particular with Fusarium spp., was not correlated with FHB resistance. The experiments demonstrate that the factor variety can influence the colonization of wheat grains by fungi. Future research must help to better understand the plant traits that govern the differential colonization. These findings should be considered in future studies on wheat storage and food safety.
\end{abstract}

Keywords: fungi, food safety, microbial ecology, wheat breeding 


\section{Introduction}

After harvest, wheat grains carry a wide range of saprophytic and pathogenic fungi. Surveys of the grain mycoflora have revealed the presence of more than 20 different fungal genera and species [1]. The occurrence of toxigenic fungi belonging to the genera Fusarium, Aspergillus, Penicillium, and Alternaria have received particular interest. The presence of these fungi raises concerns about the wholesomeness and the commercial value of the grains [2].

It is common opinion that the contamination of the grain by fungi is mainly a passive process driven by rain splash and wind moving fungal spores or eventually small parts of the hyphae to the spike [3]. Contamination may therefore occur at any moment between anthesis and storage, reflecting the composition of the mycoflora residing in the environment of the plant [4]. Environmental factors such as the presence of determined fungal species, humidity, wind, and other climatic factors also influence the colonization of grains with fungi.

The diversity and the predominance of fungal species are influenced by climatic and other environmental factors. This is best studied for the pathogens of the Fusarium head blight (FHB) complex on wheat, where crop residues constitute the principal source of inoculum in the field [5, 6]. Fusarium graminearum predominates in Central and southeastern Europe, while in cooler maritime climates mainly F. culmorum, F. avenaceum, F. poae, and Microdochium species occur [7]. Arguably, these distribution patterns are changing with changing cropping techniques. Fusarium graminearum is spreading more northward following the expansion of maize cultivation in cooler regions [8], and it is quite variable in pathogenicity. On the other hand, F. poae is increasing with respect to $F$. culmorum in all cropping areas [9]. For grain colonizers that are non- or low-pathogenic in Europe such as Penicillium spp., Aspergillus spp., and Alternaria spp., survival in soil is certainly conditioned by the presence of crop residues and other soil factors [3]. The previous crop also plays an important role in this case.

Besides the environmental conditions, morphological traits such as plant height, earliness, spike conformation, and flowering type influence the colonization of the grains. The contamination process of the grains may be in part comparable to the infection process of FHB on wheat. With fungal contamination occurring at anthesis, the fungi may grow for the first time on the palea and the lemma and spread in a second time onto the developing grain. This is well documented with pathogenic Fusarium species that colonize the spikelet tissues before infecting the floral tissue [10]. Once established, the fungi colonize the developing grain and constitute communities on the grain that reside on the surface of the grain or in the upper layers of the teguments.

The present work addresses the question of the influence of the plant on diversity and abundance of fungal grain colonizers. For this, 10 different wheat varieties were grown in one single environment. Fungal coloniz- ers were studied on native and surface sterilized grains using the classical isolation-purification-taxonomical determination procedure. Finally, the correlation between the colonization of the grains in terms of diversity, abundance, and resistance against FHB was studied.

\section{Material and Methods}

The investigation was carried on Changins and Cadenazzo field stations which belong to the Agroscope Institute (Switzerland). The experiment was conducted for two years (2008-09).

Five Polish and five Swiss commercial winter wheat cultivars were used in the experiments. According to observations of official variety trials, both resistance and susceptibility against FHB were included in the cultivar set. The cultivars are described in Table 1.

The 10 wheat varieties were planted in standardized plots of $1.5 \times 5 \mathrm{~m}$ using a precision seeding array. Seeds without seed treatment were planted on 15 October 2008 on a calcareous sandy brown soil with a loamy texture of $24.6 \%$ clay, $38.4 \%$ silt, and $31.7 \%$ sand at $\mathrm{pH} 8$. The precedent crops were rape seed (2008), wheat (2007), and maize (2006). Soil fertility status at seeding was organic matter $2.3 \mathrm{mg} \mathrm{kg}^{-1}$, phosphate $105 \mathrm{mg} \mathrm{kg}^{-1}$, potassium $157.5 \mathrm{mg} \mathrm{kg}^{-1}$, and magnesium $167.4 \mathrm{mg} \mathrm{kg}^{-1} . \mathrm{N}_{\min }$, the nitrogen level at the end of winter (February 2009), accounted for 39 units. Nitrogen fertilization consisted of two additions of Lonza-sol N liquid (Lonza AG, Basel, Switzerland) $0.155 \mathrm{t} \mathrm{ha}^{-1}$ and $0.18 \mathrm{t} \mathrm{ha}^{-1}$ before heading (corresponding to a total of 130 units N). Phosphate and

Table 1. Winter wheat varieties used in the experiment.

\begin{tabular}{|c|c|c|}
\hline Variety & Breeder & $\begin{array}{c}\text { Year of } \\
\text { registration }\end{array}$ \\
\hline Arina & $\begin{array}{c}\text { Agroscope / DSP } \\
\text { (Switzerland) }\end{array}$ & 1981 \\
\hline Runal & $\begin{array}{c}\text { Agroscope / DSP } \\
\text { (Switzerland) }\end{array}$ & 1995 \\
\hline Levis & $\begin{array}{c}\text { Agroscope / DSP } \\
\text { (Switzerland) }\end{array}$ & 1997 \\
\hline Liryka & $\begin{array}{c}\text { Malopolska Hodowla Roslin } \\
\text { (Poland) }\end{array}$ & 1998 \\
\hline Rywalka & $\begin{array}{c}\text { Hodowla Roślin Strzelce } \\
\text { sp. z o.o. - Grupa IHAR }\end{array}$ & 2000 \\
\hline Tonacja & $\begin{array}{c}\text { Hodowla Roślin Strzelce } \\
\text { sp. z o.o. - Grupa IHAR }\end{array}$ & 2001 \\
\hline Bogatka & $\begin{array}{c}\text { Danko Hodowla Roslin } \\
\text { (Poland) }\end{array}$ & 2004 \\
\hline Batuta & $\begin{array}{c}\text { Danko Hodowla Roslin } \\
\text { (Poland) }\end{array}$ & 2006 \\
\hline Claro & $\begin{array}{c}\text { Agroscope / DSP } \\
\text { (Switzerland) }\end{array}$ & 2009 \\
\hline Combin & $\begin{array}{c}\text { Agroscope / DSP } \\
\text { (Switzerland) }\end{array}$ & 2010 \\
\hline
\end{tabular}


potassium were not added. Weeds were controlled with Plüsstar (31.2\% Mecoprop-P and 18.2\% 2.4-D, Omya (Schweiz) AG, Oftringen, Switzerland) and Starane 180 (26.01\% Fluroxypyr as ester, Stähler Suisse AG, Zofingen, Switzerland) on 3 April 2009 and with Concert (68.2\% Thifensulfuraon-methyl and 6.8\% Metsulfuronmethyl, Du Pont International Operations S.A., Le GrandSacconex, Switzerland) on 14 April 2009. No insecticide or fungicide treatments were applied. On 30 January 2009 $10 \mathrm{~kg}$ of minced maize straw (variety Ceresia (DSP, Switzerland), grown 2008 in Chanigins), presenting immature apothecia of $F$. graminearum were distributed uniformly between the rows of wheat.

The crop was harvested on 20 July 2009 with a combine plots harvester (Wintersteiger AG, Ried, Austria). The yield of each plot was collected separately in clean linen sacks. After drying to $15 \%$ relative humidity, the grain was cleaned and sorted with a GrainGage stationary trial grain sorter (Juniper Systems, Logan, USA). Cleaned and conditioned grain was stored in fresh linen sacks at $+4^{\circ} \mathrm{C}$ until further use. Observations of the plot during the growing season did not reveal any symptoms of Fusarium head blight (FHB) or ear blotch (Stagonospora nodorum) on the spike. After harvest, grains showed normal shape and no shrivelled or Fusarium-damaged grains were detected.

Six-hundred grains were chosen at random from each batch. Three-hundred seeds were surface-disinfected in sodium hypochlorite ( $1 \%$ available chlorine) for $30 \mathrm{sec}$ before transfer on 2\% malt extract agar (MEA, Biocorp) in $90 \mathrm{~mm}$ Petri dishes, and the other 100 seeds were placed on $2 \%$ MEA in Petri dishes without surface disinfection. All variant experiments were performed in triplicate. The incubation of cultures on Petri dishes was carried out at room temperature $\left(22^{\circ} \mathrm{C}\right)$ for $10-14$ days in darkness. After incubation, fungi were identified on the basis of the morphology of their spores. The frequency of a single taxon (in \%) was calculated on the basis of its proportion to the total number of strains in one treatment.

The resistance against Fusarium head blight of the wheat cultivars was tested in 2010 at two trial sites in Switzerland: Changins (canton Vaud, altitude $450 \mathrm{~m}$ ) and Cadenazzo (canton Ticino, altitude $250 \mathrm{~m}$ ). The cultivars were planted in $1 \mathrm{~m}$ rows with the multiple row seeder Hege Seedmatic (Hege Maschinen, Eging am See, Germany) in Changins or in $20 \times 20 \mathrm{~cm}$ miniplots by hand in Cadenazzo. Upon flowering, plants were infected with conidiospores of F. culmorum produced on oat grains [7]. Plants were uniformly treated with a backpack sprayer (Spray Matic 10 B, Birchmeier Sprühtechnik AG, Stetten, Switzerland) using $30 \mathrm{ml}$ spore suspension per $\mathrm{m}^{2}$ at a concentration of $10^{6}$ spores per $\mathrm{ml}$. Two weeks after infection and until ripening of the plants, disease severity was assessed visually as a proportion of spikelets presenting typical head blight symptoms. Disease severity was assessed on a 1-9 logistical scale (Table 2) [11].

The results of fungal colonization tests were analyzed by ANOVA. Means were compared using Fisher's least
Table 2. Scoring scheme for the rating of severity of FHB on wheat heads.

\begin{tabular}{|c|c|}
\hline Scoring note & Disease severity (\%) \\
\hline 1 & 0.0 \\
\hline 2 & 2.5 \\
\hline 3 & 10.0 \\
\hline 4 & 25.0 \\
\hline 5 & 50.0 \\
\hline 6 & 75.0 \\
\hline 7 & 90.0 \\
\hline 8 & 98.5 \\
\hline 9 & 100.0 \\
\hline
\end{tabular}

significant difference (LSD) test at $\alpha \leq 0.01$. The number of isolates of each species was averaged and presented as a percentage of total isolates. Percentage data were transformed to Bliss angular degrees by the formula $y=\arcsin$ (value $\%)^{-0.5}$. After transformation, the variance is approximately constant, allowing analysis of variance to compare proportions of species between treatments and wheat varieties. Comparison of the presence and absence of certain fungal species has been studied using the HaGis application. Each variety was assigned a numerical designation (Gilmour Code, 9) by HaGis based on the composition of its grain mycoflora. The resulting number defines the affinity of the fungal species toward the wheat varieties. The application calculates descriptive parameters of the populations such as colonization frequency and complexity of fungal colonizers.

The resistance tests were set up as completely randomized blocks with three replicates (blocks). The mean disease severity value for each variety was calculated by averaging the disease severity values at each assessment point. The area under disease progress curve (AUDPC) was calculated using the formula $\mathrm{AUDPC}=\Sigma\left[\left(\mathrm{Y}_{i+1}+\mathrm{Y}_{i}\right) / 2\right]\left[\mathrm{t}_{i}+1-\mathrm{t}_{i}\right]$, where $\mathrm{Y}_{i}$ is disease severity at the $i^{\text {th }}$ observation, $\mathrm{n}$ is the total number of observations, and $\mathrm{t}_{i}$ is the time (days) at the $i^{\text {th }}$ observation. To compare the disease severity between the two experimental sites, the relative AUDPC (AUDPCrel) was calculated by dividing the AUDPC by the number of observation days [12]. After ascertaining that the residues of the data sets of each trial site were normally distributed (Kolmogorov Smirnov test at a $<0.02)$, statistically significant differences of AUDPCrel between varieties were tested using one-way ANOVA coupled with Fisher's least significant difference (LSD) test at $\alpha \leq 0.01$. Correlations between varietal resistance and the abundance of fungal species were tested with Pearsons correlation analysis. All statistical analyses were done with the Statistica 9.1 package (StatSoft Inc., Tulsa OK, USA). 


\section{Results and Discussion}

Overall, 18 different fungal genera or species were isolated on the grains and identified. Populations of fungi from non-disinfected and disinfected grains are shown in Tables 3 and 4, respectively. The two mycobiota consisted of the same range of species but the frequency of individual species differed. Among the eight wheat varieties, the presence and the abundance of individual species varied largely in both variants (i.e., surface disinfected and nondisinfected grains) of the experiment.
Alternaria alternata, Epicoccum nigrum, Penicillium spp., Ulocladium botrytis, and the class of non-sporulating fungi were found in each batch and in both variants of the experiment (Tables 3 and 4). The other species were not detected with all varieties. Among the mycotoxin-producing fungi, seven species of the genus Fusarium, Aspergillus Niger, and different (not further identified) Penicillium species were isolated. Botrytis cinerea, Cladosporium cladosporioides, and Phoma eupyrena are plant pathogens usually not associated with wheat. To better visualize the occurrence and the colonization of grains by Fusarium

Table 3. Fungal isolates from non-disinfected grains ( $\%$ of total isolates).

\begin{tabular}{|c|c|c|c|c|c|c|c|c|c|c|}
\hline \multirow{2}{*}{ Fungus } & \multicolumn{10}{|c|}{ Cultivars } \\
\hline & Levis & Batuta & Runal & Combin & Claro & Bogatka & Rywatka & Arina & Tonacja & Liryka \\
\hline $\begin{array}{c}\text { Alternaria } \\
\text { alternata }\end{array}$ & $\begin{array}{c}18.6 \\
a_{F}\end{array}$ & $\begin{array}{c}30.2 \\
{ }_{\mathrm{D}}\end{array}$ & $\begin{array}{c}36.2 \\
{ }^{a}{ }_{C}\end{array}$ & $\begin{array}{c}12.8 \\
{ }_{\mathrm{G}}\end{array}$ & $\begin{array}{c}12.8 \\
{ }_{\mathrm{C}}{ }_{\mathrm{G}}\end{array}$ & $\begin{array}{c}14.0 \\
\mathrm{~b} \\
\mathrm{~F}\end{array}$ & $\begin{array}{c}30.6 \\
\mathrm{a} \\
{ }_{\mathrm{D}}\end{array}$ & $\begin{array}{c}46.5 \\
{ }_{\mathrm{A}}\end{array}$ & $\begin{array}{c}36.2 \\
{ }^{\mathrm{a}}{ }_{\mathrm{C}}\end{array}$ & $\begin{array}{c}39.4 \\
\text { a } \\
\text { B }\end{array}$ \\
\hline $\begin{array}{l}\text { Aspergillus } \\
\text { niger }\end{array}$ & $\begin{array}{c}0.5 \\
\mathrm{i} \\
\mathrm{E}\end{array}$ & $\begin{array}{l}1.0 \\
\mathrm{fg} \\
\end{array}$ & $\begin{array}{l}4.3 \\
{ }^{\circ} \mathrm{C} \\
\end{array}$ & $\begin{array}{c}13.8 \\
\mathrm{~b} \\
\mathrm{~A}\end{array}$ & $\begin{array}{c}13.8 \\
\mathrm{~b} \\
\mathrm{~A}\end{array}$ & $\begin{array}{c}0.9 \\
\mathrm{hi} \\
{ }_{\mathrm{DE}}\end{array}$ & $\begin{array}{l}5.6 \\
\mathrm{~d} \\
\mathrm{~B}\end{array}$ & $\begin{array}{l}1.9 \\
\mathrm{~h} \\
{ }_{\mathrm{D}}\end{array}$ & $\begin{array}{l}0.7 \\
\mathrm{~h} \\
\mathrm{E}\end{array}$ & $\begin{array}{l}1.0 \\
\mathrm{f} \\
\mathrm{DE}\end{array}$ \\
\hline Botrytis cinerea & $\begin{array}{l}0.5 \\
\mathrm{i} \\
{ }_{\mathrm{BC}}\end{array}$ & $\begin{array}{l}1.0 \\
\mathrm{fg} \\
\mathrm{AB}\end{array}$ & $\begin{array}{l}0.4 \\
\mathrm{i} \\
{ }_{\mathrm{BC}} \\
\end{array}$ & $\begin{array}{l}0.0 \\
\mathrm{j} \\
{ }_{\mathrm{C}}\end{array}$ & $\begin{array}{l}0.0 \\
{ }_{\mathrm{ij}}{ }_{\mathrm{C}} \\
\end{array}$ & $\begin{array}{l}0.0 \\
{ }^{i}{ }_{C} \\
\end{array}$ & $\begin{array}{l}0.0 \\
\mathrm{i} \\
{ }_{\mathrm{C}} \\
\end{array}$ & $\begin{array}{l}0.0 \\
\mathrm{k}{ }_{\mathrm{C}} \\
\end{array}$ & $\begin{array}{l}0.7 \\
\mathrm{~h} \\
\mathrm{ABC} \\
\end{array}$ & $\begin{array}{l}1.5 \\
{ }_{A} \\
\end{array}$ \\
\hline $\begin{array}{l}\text { Cladosporium } \\
\text { cladosporioides }\end{array}$ & $\begin{array}{c}0.0 \\
\mathrm{i} \\
\mathrm{D} \\
\end{array}$ & $\begin{array}{l}0.7 \\
\text { fgh } \\
{ }_{C} \\
\end{array}$ & $\begin{array}{c}0.0 \\
\mathrm{i} \\
\mathrm{D} \\
\end{array}$ & $\begin{array}{c}2.1 \\
\mathrm{f} \\
\mathrm{B} \\
\end{array}$ & $\begin{array}{c}2.1 \\
\mathrm{~h} \\
\mathrm{~B}\end{array}$ & $\begin{array}{l}0.0 \\
\mathrm{i} \\
\mathrm{D} \\
\end{array}$ & $\begin{array}{c}0.0 \\
\mathrm{i} \\
\mathrm{D} \\
\end{array}$ & $\begin{array}{c}7.4 \\
\mathrm{c} \\
\mathrm{A}\end{array}$ & $\begin{array}{c}0.0 \\
\mathrm{i} \\
\mathrm{D} \\
\end{array}$ & $\begin{array}{l}0.0 \\
\mathrm{~g} \\
{ }_{\mathrm{D}} \\
\end{array}$ \\
\hline $\begin{array}{l}\text { Epicoccum } \\
\text { nigrum }\end{array}$ & $\begin{array}{c}10.5 \\
d \\
{ }_{H}\end{array}$ & $\begin{array}{c}21.9 \\
\mathrm{~b}\end{array}$ & $\begin{array}{c}24.4 \\
\mathrm{~b} \\
\text { B }\end{array}$ & $\begin{array}{c}20.2 \\
{ }^{a}{ }_{D}\end{array}$ & $\begin{array}{c}20.2 \\
{ }^{a} \\
D\end{array}$ & $\begin{array}{c}18.4 \\
\mathrm{a} \\
\mathrm{E}\end{array}$ & $\begin{array}{c}12.1 \\
{ }_{\mathrm{C}}\end{array}$ & $\begin{array}{c}16.3 \\
\mathrm{~b} \\
{ }_{\mathrm{G}}\end{array}$ & $\begin{array}{c}25.3 \\
\mathrm{~b} \\
\mathrm{~A}\end{array}$ & $\begin{array}{c}17.7 \\
{ }_{\mathrm{C}}\end{array}$ \\
\hline $\begin{array}{c}\text { Fusarium } \\
\text { avenaceum }\end{array}$ & $\begin{array}{c}13.3 \\
\mathrm{c} \\
{ }_{\mathrm{B}} \\
\end{array}$ & $\begin{array}{c}4.9 \\
\mathrm{e} \\
\mathrm{D}\end{array}$ & $\begin{array}{l}8.3 \\
{ }_{\mathrm{C}} \\
\end{array}$ & $\begin{array}{l}5.3 \\
\mathrm{f} \\
\mathrm{D} \\
\end{array}$ & $\begin{array}{c}5.3 \\
\mathrm{f} \\
\mathrm{D} \\
\end{array}$ & $\begin{array}{c}14.4 \\
\mathrm{~b} \\
\mathrm{~A} \\
\end{array}$ & $\begin{array}{l}3.9 \\
\mathrm{e} \\
\mathrm{E}\end{array}$ & $\begin{array}{l}3.8 \\
\mathrm{f} \\
\mathrm{E} \\
\end{array}$ & $\begin{array}{c}3.4 \\
\mathrm{f} \\
\mathrm{E}\end{array}$ & $\begin{array}{l}0.0 \\
\mathrm{~g} \\
\end{array}$ \\
\hline $\begin{array}{l}\text { Fusarium } \\
\text { culmorum }\end{array}$ & $\begin{array}{c}16.2 \\
\mathrm{~b} \\
\mathrm{~A}\end{array}$ & $\begin{array}{l}7.3 \\
d{ }_{B}\end{array}$ & $\begin{array}{l}4.3 \\
{ }_{\mathrm{CD}}\end{array}$ & $\begin{array}{l}5.3 \\
\mathrm{f} \\
\mathrm{C}\end{array}$ & $\begin{array}{l}5.3 \\
\mathrm{f} \\
\mathrm{C}\end{array}$ & $\begin{array}{l}7.0 \\
{ }^{\circ}{ }_{B}\end{array}$ & $\begin{array}{l}3.0 \\
\text { ef } \\
{ }_{D E}\end{array}$ & $\begin{array}{l}2.6 \\
g \\
E F\end{array}$ & $\begin{array}{l}1.4 \\
\mathrm{~g} \\
\mathrm{FG}\end{array}$ & $\begin{array}{l}1.0 \\
{ }_{G}\end{array}$ \\
\hline $\begin{array}{c}\text { Fusarium } \\
\text { graminearum }\end{array}$ & $\begin{array}{l}5.2 \\
\text { ef } \\
{ }_{B}\end{array}$ & $\begin{array}{c}0.7 \\
\text { fgh } \\
\text { EF } \\
\end{array}$ & $\begin{array}{l}1.2 \\
\mathrm{~h} \\
{ }_{\mathrm{CDE}} \\
\end{array}$ & $\begin{array}{l}5.3 \\
\mathrm{f} \\
\mathrm{B} \\
\end{array}$ & $\begin{array}{l}5.3 \\
\mathrm{f} \\
\mathrm{B} \\
\end{array}$ & $\begin{array}{c}8.8 \\
\mathrm{~d} \\
{ }_{\mathrm{A}} \\
\end{array}$ & $\begin{array}{l}1.7 \\
\mathrm{gh} \\
{ }_{\mathrm{C}} \\
\end{array}$ & $\begin{array}{c}1.6 \\
\mathrm{hi} \\
\mathrm{CD} \\
\end{array}$ & $\begin{array}{c}1.0 \\
\mathrm{gh} \\
{ }_{\mathrm{CDE}} \\
\end{array}$ & $\begin{array}{l}0.0 \\
\mathrm{~g} \\
\mathrm{G} \\
\end{array}$ \\
\hline $\begin{array}{c}\text { Fusarium } \\
\text { equiseti }\end{array}$ & $\begin{array}{l}0.5 \\
{ }^{i}{ }_{C}\end{array}$ & $\begin{array}{l}0.3 \\
\mathrm{gh} \\
{ }_{\mathrm{C}}\end{array}$ & $\begin{array}{l}0.0 \\
i \mathrm{i} \\
{ }_{D}\end{array}$ & $\begin{array}{l}0.0 \\
j \\
{ }_{D}\end{array}$ & $\begin{array}{l}0.0 \\
j \\
{ }_{D}\end{array}$ & $\begin{array}{l}1.8 \\
\mathrm{gh} \\
{ }_{\mathrm{B}}\end{array}$ & $\begin{array}{l}3.0 \\
\text { ef } \\
\text { A }\end{array}$ & $\begin{array}{l}0.0 \\
k{ }_{D}\end{array}$ & $\begin{array}{l}0.0 \\
\mathrm{i} \\
\mathrm{D}\end{array}$ & $\begin{array}{l}0.0 \\
g \\
{ }_{D}\end{array}$ \\
\hline $\begin{array}{l}\text { Fusarium } \\
\text { oxysporum }\end{array}$ & $\begin{array}{c}5.7 \\
\mathrm{e} \\
\mathrm{B} \\
\end{array}$ & $\begin{array}{l}7.3 \\
\mathrm{~d} \\
{ }_{\mathrm{A}} \\
\end{array}$ & $\begin{array}{l}0.4 \\
\mathrm{i} \\
\quad \mathrm{D} \\
\end{array}$ & $\begin{array}{l}1.1 \\
\mathrm{i} \\
{ }_{\mathrm{C}} \\
\end{array}$ & $\begin{array}{l}1.1 \\
\mathrm{i} \\
{ }_{\mathrm{C}} \\
\end{array}$ & $\begin{array}{l}1.8 \\
\mathrm{gh} \\
{ }_{\mathrm{C}} \\
\end{array}$ & $\begin{array}{l}0.0 \\
\mathrm{i} \\
\mathrm{D} \\
\end{array}$ & $\begin{array}{l}1.3 \\
\mathrm{i} \\
{ }_{\mathrm{C}} \\
\end{array}$ & $\begin{array}{c}4.1 \\
\mathrm{e} \\
\mathrm{B}\end{array}$ & $\begin{array}{c}4.9 \\
\mathrm{e} \\
\mathrm{B} \\
\end{array}$ \\
\hline Fusarium poae & $\begin{array}{l}0.0 \\
\mathrm{i}^{*} \\
\mathrm{~F}^{*}\end{array}$ & $\begin{array}{c}0.0 \\
\mathrm{~h} \\
\mathrm{E}\end{array}$ & $\begin{array}{c}0.0 \\
i \\
\mathrm{i}\end{array}$ & $\begin{array}{l}0.0 \\
j \\
E\end{array}$ & $\begin{array}{l}0.0 \\
j \\
E\end{array}$ & $\begin{array}{c}0.0 \\
\mathrm{i} \\
\mathrm{E}\end{array}$ & $\begin{array}{l}0.9 \\
\mathrm{hi} \\
\mathrm{D}\end{array}$ & $\begin{array}{l}3.8 \\
\mathrm{f} \\
{ }_{\mathrm{C}} \\
\end{array}$ & $\begin{array}{c}10.9 \\
\mathrm{c} \\
\mathrm{B}\end{array}$ & $\begin{array}{c}21.7 \\
\mathrm{~b} \\
\mathrm{~A}\end{array}$ \\
\hline $\begin{array}{c}\text { Fusarium } \\
\text { sporotrichoides }\end{array}$ & $\begin{array}{l}1.9 \\
\mathrm{~h} \\
\mathrm{D}\end{array}$ & $\begin{array}{l}0.7 \\
\text { fgh } \\
\text { E }\end{array}$ & $\begin{array}{c}2.8 \\
\mathrm{~g} \\
{ }_{\mathrm{C}}\end{array}$ & $\begin{array}{l}9.6 \\
\mathrm{~d} \\
{ }_{\mathrm{A}} \\
\end{array}$ & $\begin{array}{l}9.6 \\
\mathrm{~d} \\
{ }_{\mathrm{A}} \\
\end{array}$ & $\begin{array}{c}0.0 \\
i_{F} \\
\end{array}$ & $\begin{array}{l}0.0 \\
i_{F} \\
\end{array}$ & $\begin{array}{l}4.5 \\
\mathrm{e}_{\mathrm{B}}\end{array}$ & $\begin{array}{c}0.0 \\
\mathrm{i} \\
\mathrm{F} \\
\end{array}$ & $\begin{array}{c}0.0 \\
\mathrm{~g} \\
\mathrm{~F} \\
\end{array}$ \\
\hline $\begin{array}{l}\text { Pennicillium } \\
\text { spp. }\end{array}$ & $\begin{array}{l}3.8 \\
\mathrm{~g} \\
\quad \mathrm{D}\end{array}$ & $\begin{array}{l}5.2 \\
\mathrm{e} \\
\mathrm{BC}\end{array}$ & $\begin{array}{l}5.1 \\
d \\
{ }_{B C}\end{array}$ & $\begin{array}{c}8.5 \\
\mathrm{e} \\
\mathrm{A}\end{array}$ & $\begin{array}{c}8.5 \\
\mathrm{e} \\
\mathrm{A}\end{array}$ & $\begin{array}{l}4.4 \\
\mathrm{f} \\
{ }_{\mathrm{CD}} \\
\end{array}$ & $\begin{array}{l}6.0 \\
\mathrm{~d} \\
{ }_{B}\end{array}$ & $\begin{array}{c}0.6 \\
j \\
E\end{array}$ & $\begin{array}{c}0.0 \\
\mathrm{i} \\
\mathrm{E}\end{array}$ & $\begin{array}{c}0.0 \\
g \\
E\end{array}$ \\
\hline $\begin{array}{c}\text { Phoma } \\
\text { eupyrena }\end{array}$ & $\begin{array}{l}1.9 \\
\mathrm{~h} \\
{ }_{\mathrm{A}}\end{array}$ & $\begin{array}{l}0.3 \\
\mathrm{gh} \\
{ }_{\mathrm{CD}} \\
\end{array}$ & $\begin{array}{c}0,4 \\
\mathrm{i} \\
\mathrm{C} \\
\end{array}$ & $\begin{array}{l}1.1 \\
\mathrm{i} \\
\mathrm{B} \\
\end{array}$ & $\begin{array}{l}1.1 \\
\mathrm{i} \\
\mathrm{B} \\
\end{array}$ & $\begin{array}{l}0.9 \\
\mathrm{hi} \\
\mathrm{B} \\
\end{array}$ & $\begin{array}{c}0.0 \\
\mathrm{i} \\
\mathrm{D} \\
\end{array}$ & $\begin{array}{l}0.0 \\
\mathrm{k} \\
\quad \mathrm{D} \\
\end{array}$ & $\begin{array}{l}0.0 \\
\mathrm{i} \\
\mathrm{D}\end{array}$ & $\begin{array}{c}0.0 \\
\mathrm{~g} \\
{ }_{\mathrm{D}} \\
\end{array}$ \\
\hline $\begin{array}{c}\text { Rhizopus } \\
\text { arrhizus }\end{array}$ & $\begin{array}{l}4.8 \\
\mathrm{f} \\
\mathrm{B} \\
\end{array}$ & $\begin{array}{l}1.4 \\
f \\
D \\
\end{array}$ & $\begin{array}{c}2,4 \\
g \\
{ }_{C}\end{array}$ & $\begin{array}{c}3.2 \\
g \\
B\end{array}$ & $\begin{array}{c}3.2 \\
\mathrm{~b} \\
\mathrm{~B} \\
\end{array}$ & $\begin{array}{l}3.5 \\
\mathrm{f} \\
\mathrm{B} \\
\end{array}$ & $\begin{array}{l}2.6 \\
\mathrm{fg} \\
{ }_{\mathrm{C}}\end{array}$ & $\begin{array}{c}0.0 \\
\mathrm{k} \\
\mathrm{E}\end{array}$ & $\begin{array}{c}0.0 \\
i \\
E\end{array}$ & $\begin{array}{c}0.0 \\
g \\
\end{array}$ \\
\hline $\begin{array}{l}\text { Trichoderma } \\
\text { spp. }\end{array}$ & $\begin{array}{l}4.8 \\
\mathrm{f} \\
\mathrm{A}\end{array}$ & $\begin{array}{c}0.0 \\
\mathrm{~h} \\
\mathrm{E}\end{array}$ & $\begin{array}{l}3,5 \\
f \\
\text { B }\end{array}$ & $\begin{array}{c}0.0 \\
j \\
{ }_{E}\end{array}$ & $\begin{array}{c}0.0 \\
j \\
{ }_{E}\end{array}$ & $\begin{array}{l}2.5 \\
\mathrm{~g} \\
\end{array}$ & $\begin{array}{c}0.0 \\
\mathrm{i} \\
\mathrm{E}\end{array}$ & $\begin{array}{l}1.9 \\
\mathrm{~h} \\
\mathrm{D}\end{array}$ & $\begin{array}{c}0.0 \\
\mathrm{i} \\
\mathrm{E}\end{array}$ & $\begin{array}{c}0.0 \\
\mathrm{~g} \\
\mathrm{E}\end{array}$ \\
\hline $\begin{array}{c}\text { Ulocladium } \\
\text { botrytis }\end{array}$ & $\begin{array}{c}10.5 \\
\mathrm{~d} \\
{ }_{B}\end{array}$ & $\begin{array}{c}12.5 \\
{ }_{\mathrm{A}} \\
\end{array}$ & $\begin{array}{c}3.5 \\
\mathrm{f} \\
\mathrm{F}\end{array}$ & $\begin{array}{l}9.6 \\
d \\
{ }_{C}\end{array}$ & $\begin{array}{l}9.6 \\
\mathrm{~d} \\
{ }_{C}\end{array}$ & $\begin{array}{c}8.8 \\
\mathrm{~d} \\
\mathrm{D} \\
\end{array}$ & $\begin{array}{c}3.4 \\
\text { ef } \\
\quad F\end{array}$ & $\begin{array}{c}2.7 \\
g \\
{ }_{G}\end{array}$ & $\begin{array}{c}4.8 \\
\mathrm{~d} \\
\mathrm{E}\end{array}$ & $\begin{array}{c}1.5 \\
\mathrm{f} \\
\mathrm{H} \\
\end{array}$ \\
\hline $\begin{array}{c}\text { Non-sporulating } \\
\text { fungi }\end{array}$ & $\begin{array}{c}1.3 \\
\mathrm{~h} \\
\mathrm{H}\end{array}$ & $\begin{array}{c}4.6 \\
e \\
E\end{array}$ & $\begin{array}{c}2.8 \\
\mathrm{~g} \\
\mathrm{~F}\end{array}$ & $\begin{array}{l}2.1 \\
\mathrm{~h} \\
{ }_{\mathrm{G}}\end{array}$ & $\begin{array}{l}2.1 \\
\mathrm{~h} \\
{ }_{\mathrm{G}}\end{array}$ & $\begin{array}{c}12.8 \\
{ }_{\mathrm{B}} \\
\end{array}$ & $\begin{array}{c}27.2 \\
\mathrm{~b} \\
\mathrm{~A} \\
\end{array}$ & $\begin{array}{l}5.1 \\
d{ }_{D}\end{array}$ & $\begin{array}{c}11.5 \\
{ }^{c} \\
\end{array}$ & $\begin{array}{c}11.3 \\
\mathrm{~d} \\
{ }_{C}\end{array}$ \\
\hline
\end{tabular}

*Means followed by the same letter do not differ significantly. Small letters mark the effect of of individual cultivars on isolates fungi from grain; they refer to means in columns. Capital letters mark the effects of cultivars on individual species of fungi; they refer to means in rows. Fisher's least significant difference (LSD) test, $\alpha \leq 0.01$. 
species on each wheat variety, data on the presence/absence were generated with the application HaGis (Fig. 1).

The varieties Bogatka and Tonacja were colonized by the same Fusarium species while the other varieties presented all different patterns of colonization. A low similarity $(<50)$ was detected between Claro and Levis, Claro and Batuta, Lyrika and Batuta, and Liryka and Levis. High similarity $(>80)$ was detected between Batuta and Levis, Batuta and Bogatka/Tonacja, Runal and Batuta, and Rywatka and Bogatka/Tonacja. The other combinations showed intermediate similarity (between 50 and 80 ).
The proportions of fungal species isolated from the grains of each variety are presented in Tables 3 and 4 for surface non-disinfected and surface-disinfected grains, respectively. Alternaria alternata was the most abundant species on all wheat varieties from the surface and inside the teguments of grains. The proportion of grains with A. alternata was similar in Levis, Runal, Rywatka, and Tonacja (about 20 to $35 \%$ ). Smallest frequencies were recorded on Batuta (7.3\%), Combin (3.1\%), and Bogatka (1.9\%). Epicoccum nigrum was the second most abundant species found. The frequencies of E. nigrum amounted to

Table 4. Fungal isolates from disinfected grains ( $\%$ of total isolates).

\begin{tabular}{|c|c|c|c|c|c|c|c|c|c|c|}
\hline \multirow{2}{*}{ Fungus } & \multicolumn{10}{|c|}{ Cultivars } \\
\hline & Levis & Batuta & Runal & Combin & Claro & Bogatka & Rywatka & Arina & Tonacja & Liryka \\
\hline $\begin{array}{c}\text { Alternaria } \\
\text { alternata }\end{array}$ & $\begin{array}{l}24.9 \\
\mathrm{a}^{*} \\
\mathrm{E}^{*}\end{array}$ & $\begin{array}{l}7.3 \\
\mathrm{~d} \\
\mathrm{H}\end{array}$ & $\begin{array}{c}35.1 \\
{ }_{\mathrm{A}}\end{array}$ & $\begin{array}{c}3.1 \\
i \\
1\end{array}$ & $\begin{array}{c}30.2 \\
{ }^{\mathrm{a}}{ }_{\mathrm{C}}\end{array}$ & $\begin{array}{c}1.9 \\
\mathrm{i} \\
\mathrm{J}\end{array}$ & $\begin{array}{c}31.4 \\
{ }^{\mathrm{a}}{ }_{\mathrm{B}}\end{array}$ & $\begin{array}{c}19.1 \\
a_{F}\end{array}$ & $\begin{array}{c}28.0 \\
{ }^{a}{ }_{D}\end{array}$ & $\begin{array}{c}15.4 \\
{ }_{\mathrm{G}}\end{array}$ \\
\hline Aspergillus niger & $\begin{array}{l}2.0 \\
\mathrm{gh} \\
\mathrm{B}\end{array}$ & $\begin{array}{l}0.0 \\
\mathrm{k} \\
{ }_{\mathrm{D}}\end{array}$ & $\begin{array}{l}1.7 \\
\mathrm{f} \\
{ }_{\mathrm{BC}} \\
\end{array}$ & $\begin{array}{c}10.2 \\
\mathrm{~d} \\
\mathrm{~A}\end{array}$ & $\begin{array}{l}1.9 \\
\mathrm{~h} \\
{ }_{\mathrm{BC}} \\
\end{array}$ & $\begin{array}{l}0.0 \\
\mathrm{k} \\
{ }_{\mathrm{D}} \\
\end{array}$ & $\begin{array}{l}1.5 \\
\mathrm{f} \\
{ }_{\mathrm{C}} \\
\end{array}$ & $\begin{array}{l}0.0 \\
\mathrm{j} \\
\end{array}$ & $\begin{array}{l}0.0 \\
j \\
{ }_{D}\end{array}$ & $\begin{array}{l}0.0 \\
\mathrm{~h} \\
\mathrm{D} \\
\end{array}$ \\
\hline Botrytis cinerea & $\begin{array}{l}1.5 \\
{ }_{\mathrm{h}}\end{array}$ & $\begin{array}{l}0.0 \\
{ }_{\mathrm{k}}{ }_{\mathrm{C}}\end{array}$ & $\begin{array}{l}0.8 \\
\mathrm{~g} \\
{ }_{B}\end{array}$ & $\begin{array}{l}0.0 \\
1 \\
{ }_{C}\end{array}$ & $\begin{array}{l}0.0 \\
{ }^{j}\end{array}$ & $\begin{array}{l}0.0 \\
{ }_{\mathrm{k}}{ }_{\mathrm{C}}\end{array}$ & $\begin{array}{l}0.0 \\
{ }_{\mathrm{h}}{ }_{\mathrm{C}}\end{array}$ & $\begin{array}{l}0.0 \\
{ }^{j}\end{array}$ & $\begin{array}{l}0.0 \\
{ }_{\mathrm{j}}\end{array}$ & $\begin{array}{l}0.0 \\
{ }_{\mathrm{h}}{ }_{\mathrm{C}}\end{array}$ \\
\hline $\begin{array}{l}\text { Cladosporium } \\
\text { cladosporioides }\end{array}$ & $\begin{array}{c}0.0 \\
\mathrm{i} \\
\mathrm{F}\end{array}$ & $\begin{array}{l}4.2 \\
\mathrm{~g} \\
\quad \mathrm{~B} \\
\end{array}$ & $\begin{array}{l}0.4 \\
\mathrm{~g} \\
\mathrm{EF}\end{array}$ & $\begin{array}{l}1.6 \\
\mathrm{j} \\
{ }_{\mathrm{C}}\end{array}$ & $\begin{array}{l}0.9 \\
\mathrm{i} \\
\mathrm{DE}\end{array}$ & $\begin{array}{c}0.0 \\
{ }_{\mathrm{F}} \\
\end{array}$ & $\begin{array}{l}0.0 \\
\mathrm{~h} \\
{ }_{\mathrm{F}}\end{array}$ & $\begin{array}{c}13.5 \\
\mathrm{~b} \\
\mathrm{~A} \\
\end{array}$ & $\begin{array}{c}1.4 \\
\mathrm{hi} \\
\quad \mathrm{CD} \\
\end{array}$ & $\begin{array}{l}1.6 \\
\mathrm{~g} \\
{ }_{\mathrm{C}}\end{array}$ \\
\hline $\begin{array}{l}\text { Epicoccum } \\
\text { nigrum }\end{array}$ & $\begin{array}{c}23.4 \\
\mathrm{~b} \\
\mathrm{~F} \\
\end{array}$ & $\begin{array}{c}32.5 \\
\mathrm{a} \\
{ }_{\mathrm{A}} \\
\end{array}$ & $\begin{array}{c}27.3 \\
\mathrm{~b} \\
{ }_{\mathrm{C}} \\
\end{array}$ & $\begin{array}{c}10.9 \\
c \\
{ }_{I} \\
\end{array}$ & $\begin{array}{c}26.4 \\
\mathrm{~b} \\
\mathrm{D}\end{array}$ & $\begin{array}{c}9.3 \\
\mathrm{~d} \\
\mathrm{~J}\end{array}$ & $\begin{array}{c}28.5 \\
\mathrm{~b} \\
\mathrm{~B} \\
\end{array}$ & $\begin{array}{c}12.1 \\
\mathrm{~b} \\
\mathrm{H}\end{array}$ & $\begin{array}{c}21.5 \\
\mathrm{~b} \\
{ }_{\mathrm{G}} \\
\end{array}$ & $\begin{array}{c}25.2 \\
\mathrm{~b} \\
\mathrm{E}\end{array}$ \\
\hline $\begin{array}{c}\text { Fusarium } \\
\text { avenaceum }\end{array}$ & $\begin{array}{c}10.1 \\
\mathrm{c} \\
\mathrm{D}\end{array}$ & $\begin{array}{c}7.9 \\
c_{F} \\
\end{array}$ & $\begin{array}{l}3.7 \\
\mathrm{e} \\
{ }_{\mathrm{G}}\end{array}$ & $\begin{array}{c}14.1 \\
\mathrm{~b} \\
\mathrm{~A}\end{array}$ & $\begin{array}{c}13.2 \\
\mathrm{c} \\
{ }_{\mathrm{B}}\end{array}$ & $\begin{array}{c}7.5 \\
e \\
\quad F\end{array}$ & $\begin{array}{l}2.9 \\
\mathrm{e} \\
\mathrm{H}\end{array}$ & $\begin{array}{c}9.2 \\
{ }_{\mathrm{E}} \\
\end{array}$ & $\begin{array}{c}10.7 \\
{ }_{\mathrm{C}} \\
\end{array}$ & $\begin{array}{c}0.0 \\
\mathrm{~h} \\
\mathrm{I}\end{array}$ \\
\hline $\begin{array}{l}\text { Fusarium } \\
\text { culmorum }\end{array}$ & $\begin{array}{l}5.1 \\
\mathrm{e} \\
{ }_{\mathrm{C}}\end{array}$ & $\begin{array}{c}2.1 \\
\mathrm{i} \\
\mathrm{F}\end{array}$ & $\begin{array}{l}5.0 \\
{ }_{C} \\
\end{array}$ & $\begin{array}{c}9.4 \\
\mathrm{e} \\
\mathrm{A}\end{array}$ & $\begin{array}{l}4.7 \\
{ }^{\prime}{ }_{C D}\end{array}$ & $\begin{array}{l}4.7 \\
\mathrm{~g} \\
\mathrm{CD}\end{array}$ & $\begin{array}{l}0.7 \\
\mathrm{gh} \\
{ }_{\mathrm{G}}\end{array}$ & $\begin{array}{c}6.4 \\
\mathrm{e} \\
\mathrm{B}\end{array}$ & $\begin{array}{c}3.1 \\
f \\
E\end{array}$ & $\begin{array}{c}4.1 \\
\mathrm{e} \\
\mathrm{D}\end{array}$ \\
\hline $\begin{array}{c}\text { Fusarium } \\
\text { graminearum }\end{array}$ & $\begin{array}{l}2.5 \\
g \\
{ }_{C D} \\
\end{array}$ & $\begin{array}{l}2.1 \\
\mathrm{i} \\
\mathrm{DE} \\
\end{array}$ & $\begin{array}{l}1.7 \\
\mathrm{f} \\
\mathrm{E}\end{array}$ & $\begin{array}{l}4.7 \\
\mathrm{~h} \\
\mathrm{~B}\end{array}$ & $\begin{array}{l}0.0 \\
j \\
\end{array}$ & $\begin{array}{l}2.8 \\
\mathrm{~h} \\
{ }_{\mathrm{C}} \\
\end{array}$ & $\begin{array}{c}2.9 \\
\mathrm{e} \\
{ }_{\mathrm{C}} \\
\end{array}$ & $\begin{array}{l}0.0 \\
j \\
F\end{array}$ & $\begin{array}{l}5.9 \\
\mathrm{e}_{\mathrm{A}}\end{array}$ & $\begin{array}{l}0.0 \\
\mathrm{~h} \\
\mathrm{~F}\end{array}$ \\
\hline $\begin{array}{c}\text { Fusarium } \\
\text { equiseti }\end{array}$ & $\begin{array}{l}2.0 \\
\mathrm{gh} \\
{ }_{\mathrm{C}}\end{array}$ & $\begin{array}{l}3.1 \\
\mathrm{~h} \\
{ }_{\mathrm{B}} \\
\end{array}$ & $\begin{array}{l}0.8 \\
\mathrm{~g} \\
{ }_{\mathrm{D}}\end{array}$ & $\begin{array}{l}0.8 \\
\mathrm{k} \\
\quad \mathrm{D}\end{array}$ & $\begin{array}{l}0.0 \\
j \\
{ }_{D}\end{array}$ & $\begin{array}{c}15.0 \\
c \\
{ }_{A} \\
\end{array}$ & $\begin{array}{l}0.0 \\
\mathrm{~h} \\
\mathrm{D}\end{array}$ & $\begin{array}{l}2.1 \\
\mathrm{~h} \\
\mathrm{C}_{\mathrm{C}}\end{array}$ & $\begin{array}{l}2.8 \\
\mathrm{fg} \\
{ }_{\mathrm{BC}}\end{array}$ & $\begin{array}{l}0.0 \\
\mathrm{~h} \\
{ }_{\mathrm{D}}\end{array}$ \\
\hline $\begin{array}{l}\text { Fusarium } \\
\text { oxysporum }\end{array}$ & $\begin{array}{c}10.2 \\
{ }_{\mathrm{A}}\end{array}$ & $\begin{array}{c}6.3 \\
\mathrm{e} \\
\mathrm{B}\end{array}$ & $\begin{array}{l}2.5 \\
\mathrm{f} \\
\mathrm{D}\end{array}$ & $\begin{array}{c}0.8 \\
{ }_{\mathrm{E}}\end{array}$ & $\begin{array}{l}0.0 \\
j \\
{ }_{F}\end{array}$ & $\begin{array}{c}0.0 \\
\mathrm{k} \\
\mathrm{F}\end{array}$ & $\begin{array}{c}0.0 \\
\mathrm{~h} \\
\mathrm{~F}\end{array}$ & $\begin{array}{l}3.5 \\
{ }_{\mathrm{g}}\end{array}$ & $\begin{array}{l}0.7 \\
\mathrm{ij} \\
\mathrm{E}\end{array}$ & $\begin{array}{l}0.0 \\
\mathrm{~h} \\
\mathrm{~F}\end{array}$ \\
\hline Fusarium poae & $\begin{array}{c}0.0 \\
\mathrm{i} \\
\mathrm{H}\end{array}$ & $\begin{array}{l}0.0 \\
\mathrm{k} \\
\mathrm{H}\end{array}$ & $\begin{array}{l}0.0 \\
g_{H}\end{array}$ & $\begin{array}{c}16.4 \\
\mathrm{a} \\
\mathrm{A}\end{array}$ & $\begin{array}{c}9.5 \\
\mathrm{~d} \\
{ }_{B}\end{array}$ & $\begin{array}{l}5.6 \\
f \\
D\end{array}$ & $\begin{array}{l}3.6 \\
\text { de } \\
\end{array}$ & $\begin{array}{l}7.1 \\
{ }_{C} \\
\end{array}$ & $\begin{array}{l}2.1 \\
\mathrm{gh} \\
{ }_{\mathrm{G}}\end{array}$ & $\begin{array}{c}4.9 \\
d_{E} \\
\end{array}$ \\
\hline $\begin{array}{c}\text { Fusarium } \\
\text { sporotrichoides }\end{array}$ & $\begin{array}{c}0.5 \\
\mathrm{i} \\
\mathrm{D}\end{array}$ & $\begin{array}{l}1.6 \\
j \\
{ }_{C}\end{array}$ & $\begin{array}{c}0.0 \\
g \\
E\end{array}$ & $\begin{array}{c}8.6 \\
\mathrm{f} \\
\mathrm{A}\end{array}$ & $\begin{array}{c}0.0 \\
j \\
E\end{array}$ & $\begin{array}{c}0.0 \\
\mathrm{k} \\
\mathrm{E}\end{array}$ & $\begin{array}{c}0.0 \\
\mathrm{~h} \\
\mathrm{E}\end{array}$ & $\begin{array}{c}2.1 \\
\mathrm{~h} \\
\mathrm{~B}\end{array}$ & $\begin{array}{l}0.7 \\
{ }_{\mathrm{ij}} \\
\end{array}$ & $\begin{array}{l}0.0 \\
\mathrm{~h} \\
\mathrm{E}\end{array}$ \\
\hline Pennicillium spp. & $\begin{array}{l}1.5 \\
\mathrm{~h} \\
\mathrm{DE}\end{array}$ & $\begin{array}{l}3.1 \\
\mathrm{~h} \\
\mathrm{BC} \\
\end{array}$ & $\begin{array}{l}2.5 \\
\mathrm{f} \\
{ }_{\mathrm{CD}}\end{array}$ & $\begin{array}{l}3.1 \\
\mathrm{i} \\
\mathrm{BC}\end{array}$ & $\begin{array}{l}3.8 \\
\mathrm{~g} \\
\quad \mathrm{~B} \\
\end{array}$ & $\begin{array}{l}7.5 \\
\mathrm{e} \\
\mathrm{A}\end{array}$ & $\begin{array}{c}4.0 \\
d \\
B\end{array}$ & $\begin{array}{c}0.7 \\
\mathrm{i} \\
\mathrm{E} \\
\end{array}$ & $\begin{array}{c}0.7 \\
\mathrm{ij} \\
{ }_{\mathrm{E}}\end{array}$ & $\begin{array}{l}2.4 \\
\mathrm{f} \\
{ }_{\mathrm{CD}} \\
\end{array}$ \\
\hline $\begin{array}{c}\text { Phoma eupyrena } \\
\text { Sacc. }\end{array}$ & $\begin{array}{c}0.5 \\
\mathrm{i} \\
\mathrm{B} \\
\end{array}$ & $\begin{array}{c}4.2 \\
\mathrm{~g} \\
\mathrm{~A}\end{array}$ & $\begin{array}{l}0.4 \\
\mathrm{~g} \\
\mathrm{BC}\end{array}$ & $\begin{array}{l}0.0 \\
1 \\
{ }_{C}\end{array}$ & $\begin{array}{l}0.0 \\
{ }_{C} \\
\end{array}$ & $\begin{array}{l}0.0 \\
\mathrm{k} \\
{ }_{\mathrm{C}}\end{array}$ & $\begin{array}{l}0.0 \\
\mathrm{~h} \\
{ }_{\mathrm{C}}\end{array}$ & $\begin{array}{l}0.0 \\
{ }_{C} \\
C\end{array}$ & $\begin{array}{l}0.0 \\
{ }^{j} \\
\end{array}$ & $\begin{array}{l}0.0 \\
\mathrm{~h} \\
{ }_{\mathrm{C}}\end{array}$ \\
\hline $\begin{array}{l}\text { Rhizopus } \\
\text { arrhizus }\end{array}$ & $\begin{array}{c}0.0 \\
\mathrm{i} \\
\mathrm{C} \\
\end{array}$ & $\begin{array}{c}5.2 \\
\mathrm{f} \\
\mathrm{B}\end{array}$ & $\begin{array}{l}0.0 \\
\mathrm{~g} \\
{ }_{\mathrm{C}}\end{array}$ & $\begin{array}{l}0.0 \\
1 \\
\mathrm{C} \\
\end{array}$ & $\begin{array}{l}0.0 \\
{ }_{\mathrm{j}} \\
\mathrm{C}\end{array}$ & $\begin{array}{c}22.4 \\
\mathrm{a} \\
\mathrm{A} \\
\end{array}$ & $\begin{array}{l}0.0 \\
\mathrm{~h} \\
{ }_{\mathrm{C}}\end{array}$ & $\begin{array}{l}0.0 \\
\mathrm{j} \\
{ }_{\mathrm{C}} \\
\end{array}$ & $\begin{array}{l}0.0 \\
\mathrm{j} \\
{ }_{\mathrm{C}} \\
\end{array}$ & $\begin{array}{l}0.0 \\
\mathrm{~h} \\
{ }_{\mathrm{C}} \\
\end{array}$ \\
\hline Trichoderma spp. & $\begin{array}{l}3.6 \\
\mathrm{f} \\
\mathrm{A}\end{array}$ & $\begin{array}{c}2.1 \\
\mathrm{i} \\
\mathrm{B}\end{array}$ & $\begin{array}{c}2.5 \\
\mathrm{f} \\
\mathrm{B} \\
\end{array}$ & $\begin{array}{c}0.0 \\
1 \\
\mathrm{D} \\
\end{array}$ & $\begin{array}{c}0.0 \\
\mathrm{j} \\
{ }_{\mathrm{D}}\end{array}$ & $\begin{array}{l}0.9 \\
\mathrm{j} \\
{ }_{C} \\
\end{array}$ & $\begin{array}{l}0.8 \\
\mathrm{fg} \\
{ }_{\mathrm{C}} \\
\end{array}$ & $\begin{array}{l}0.0 \\
\mathrm{j} \\
\mathrm{D}\end{array}$ & $\begin{array}{l}0.0 \\
\mathrm{j} \\
{ }_{\mathrm{D}} \\
\end{array}$ & $\begin{array}{l}0.0 \\
\mathrm{~h} \\
{ }_{\mathrm{D}}\end{array}$ \\
\hline $\begin{array}{c}\text { Ulocladium } \\
\text { botrytis }\end{array}$ & $\begin{array}{l}8.6 \\
d{ }_{B} \\
\end{array}$ & $\begin{array}{l}4.2 \\
\mathrm{~g} \\
{ }_{\mathrm{D}}\end{array}$ & $\begin{array}{l}2.5 \\
\mathrm{f} \\
\mathrm{FE}\end{array}$ & $\begin{array}{l}7.8 \\
\mathrm{~g} \\
\mathrm{~B}\end{array}$ & $\begin{array}{c}1.9 \\
\mathrm{~h} \\
\mathrm{~F}\end{array}$ & $\begin{array}{l}5.6 \\
\mathrm{f} \\
\mathrm{C}\end{array}$ & $\begin{array}{c}2.9 \\
\mathrm{e} \\
\mathrm{E}\end{array}$ & $\begin{array}{c}4.7 \\
f \\
D \\
\end{array}$ & $\begin{array}{c}10.0 \\
\mathrm{~d} \\
\mathrm{~A}\end{array}$ & $\begin{array}{l}4.9 \\
\mathrm{~d} \\
{ }_{\mathrm{CD}}\end{array}$ \\
\hline $\begin{array}{l}\text { Non-sporulating } \\
\text { fungi }\end{array}$ & $\begin{array}{c}3.6 \\
\mathrm{f} \\
\mathrm{I} \\
\end{array}$ & $\begin{array}{c}14.1 \\
b \\
{ }_{E} \\
\end{array}$ & $\begin{array}{c}13.2 \\
\mathrm{c} \\
\mathrm{F}\end{array}$ & $\begin{array}{l}8.6 \\
f \\
G\end{array}$ & $\begin{array}{c}7.5 \\
\mathrm{e} \\
\mathrm{H}\end{array}$ & $\begin{array}{c}16.8 \\
\mathrm{~b} \\
\mathrm{D} \\
\end{array}$ & $\begin{array}{c}20.8 \\
\mathrm{c} \\
{ }_{B} \\
\end{array}$ & $\begin{array}{c}19.5 \\
{ }^{\mathrm{a}}{ }_{\mathrm{C}} \\
\end{array}$ & $\begin{array}{c}12.5 \\
\mathrm{c} \\
\mathrm{F}\end{array}$ & $\begin{array}{c}41.5 \\
\mathrm{a} \\
{ }_{\mathrm{A}} \\
\end{array}$ \\
\hline
\end{tabular}

*Means followed by the same letter do not differ significantly. Small letters mark the effect of individual cultivars on isolates fungi from grain; they refer to means in columns. Capital letters mark the effects of cultivars on individual species of fungi; they refer to means in rows. Fisher's least significant difference (LSD) test, $\alpha \leq 0.01$. 


\begin{tabular}{|c|c|c|c|c|c|c|c|c|c|}
\hline Varieties & $\frac{\circ}{\frac{O}{0}}$ & 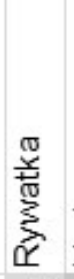 & 感 & 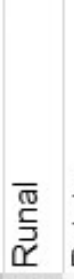 & 苑 & 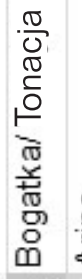 & 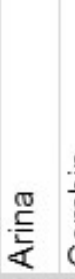 & $\begin{array}{l}\text { 등 } \\
\text { E⿱ } \\
\text { 잉 }\end{array}$ & $\stackrel{\frac{\omega}{J}}{\supset}$ \\
\hline Claro & & 57 & 67 & 57 & 44 & 67 & 60 & 60 & 40 \\
\hline Rywatka & & & 57 & 50 & 60 & 80 & 55 & 73 & 55 \\
\hline Liryka & & & & 57 & 44 & 67 & 60 & 60 & 40 \\
\hline Runal & & & & & 80 & 60 & 73 & 55 & 73 \\
\hline Batuta & & & & & & 83 & 77 & 62 & 92 \\
\hline Bogatka /Tonac & & & & & & & 77 & 77 & 77 \\
\hline Arina & & & & & & & & 71 & 71 \\
\hline Combin & & & & & & & & & 71 \\
\hline Levis & & & & & & & & & \\
\hline & & & & & & & & & \\
\hline Similarity: & & & & & & & & & \\
\hline Sample average: & 68 & & & & & & & & \\
\hline Sample Std.dev.: & 18 & & & & & & & & \\
\hline
\end{tabular}

Fig. 1. Representation of the colonization of the grains of the 10 wheat varieties by Fusarium species using the HaGis approach.

9.3-32.5\%, and there was no difference between colonization on the surface and inside the teguments. The frequencies of Fusarium spp. averaged between 2.5 and $12.5 \%$ on non-disinfected seeds and between $9.0-54.8 \%$ for surfacedisinfected seeds. Fusarium avenaceum was particularly common on both non-disinfected and disinfected grains, and numbers of colonies were similar for Levis, Batuta, Combin, Rywatka, Arina, and Tonacja. This fungus was almost absent on the variety Claro and not at all isolated on the variety Liryka (Table 3 ). Other fungi - including Penicillium, Botrytis, Trichoderma, and Phoma - occurred sporadically.

The results obtained in the first part of this study suggest an interaction between the colonization of grains by

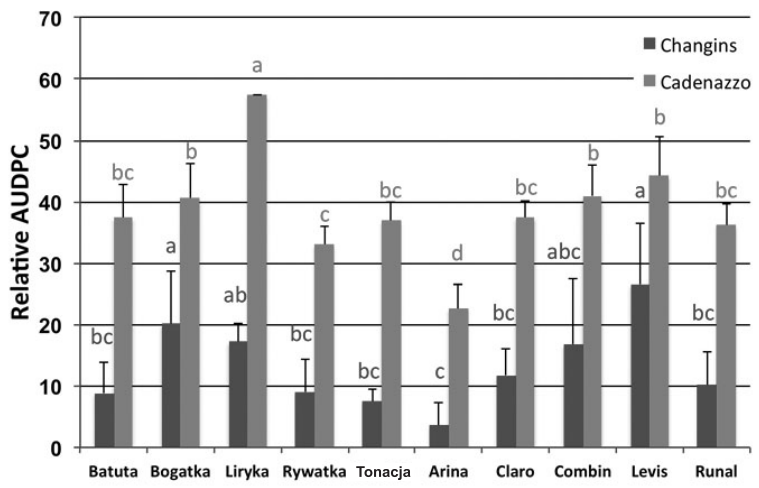

Fig. 2. Reaction of winter wheat varieties toward Fusarium head blight expressed as an area under disease progress curve (AUDPC). Letters on the top of the columns indicate statistically significant differences $(\mathrm{P}<0.05)$.
Fusarium and the wheat variety. Therefore, the second part aimed at characterizing the resistance of the varieties against FHB infections in order to compare varietal resistance and grain colonization. The tests were realized with artificial inoculations at two trial sites. Disease severity was integrated with duration of observation to obtain the AUDPC (Fig. 2).

Results indicate that Arina was the most resistant variety in both sites, while the most susceptible variety was Liryka in Cadenazzo and Levis in Changins. All other varieties showed intermediate resistance in both sites. Correlation analysis did not yield any evidence for an eventual correlation between resistance to FHB and grain colonization (Table 5).

Both fungal diversity and abundance revealed large differences between the wheat varieties. Not all fungal species have colonized all varieties. All types of fungi were concerned, but this observation was particularly evident with Fusarium species. Semaskiene et al. [13] reported similar observations. That study reported significant differences in colonization of wheat grains. The present study adopted a systematic experimental approach with three replicates for each variety and a seminatural inoculation of the plots with maize straw, as well as standardized harvest and post-harvest processing. The protocol was aimed at avoiding any fortuitous contamination of the grain during and after harvest and during storage of the grains.

Alternaria alternata, Cladosoporium cladospoirioides, Epicoccum nigrum, and Pencillium spp. are present in all genotypes. These results agree with observations of Pusz [1], who reported that Alternaria species (including A. alternata) are the most common colonizers of grains. In general, Alternaria spp. can be regarded as ubiquitous grain colonizers [14] and indicate the baseline contamination of the grains.

Table 5. Correlation analysis between the symptoms in field (FHB) and the colonization of the grains of the 10 wheat varieties by Fusarium species.

\begin{tabular}{|c|c|c|c|c|}
\hline \multirow[b]{2}{*}{ Cadenazzo } & \multicolumn{2}{|c|}{ Changins } & \multicolumn{2}{|c|}{ Cadenazzo } \\
\hline & 0.68 & $*$ & $-/-$ & $-/-$ \\
\hline Alternaria alternata & -0.30 & $\mathrm{n} . \mathrm{s}$ & -0.24 & n.s \\
\hline Aspergillus niger & 0.26 & n.s & 0.08 & n.s \\
\hline Fusarium avenaceum & 0.06 & n.s & -0.32 & n.s \\
\hline Fusarium culmorum & 0.27 & n.s & 0.01 & n.s \\
\hline $\begin{array}{c}\text { Fusarium } \\
\text { graminearum }\end{array}$ & 0.29 & n.s & -0.00 & n.s \\
\hline Fusarium equiseti & 0.32 & n.s & -0.05 & n.s \\
\hline Fusarium oxysporum & 0.09 & n.s & -0.02 & n.s \\
\hline Fusarium poae & 0.03 & n.s & -0.024 & n.s \\
\hline $\begin{array}{c}\text { Fusarium } \\
\text { sporotrichoides }\end{array}$ & 0.04 & n.s & -0.07 & n.s \\
\hline
\end{tabular}


The large differences in the abundance of colonizers between genotypes are striking. The experimental protocol adopted drastically reduces the impact of environmental factors and supports the hypotheses that plant traits play a role in grain colonization. It is known that for Fusarium head blight, plant height and internode distance are positively correlated with disease resistance [15]. When looking at spike traits, the role of anther extrusion in the occurrence of FHB is discussed controversially. Skinnes et al. [16] report that varieties that extrude and shed the anthers show lower susceptibility to FHB. Varieties used here presented reduced anther extrusion. This trait was not correlated to grain colonization or FHB resistance (results not shown). However, even little differences in anther extrusion can have a significant impact on the susceptibility of the variety [17]. In a future investigation, the role of anthers on mycoflora of grains has to be considered.

The least contaminated grains by Fusarium spp. were found on Liryka (on disinfected grains) and Rywatka (on non-disinfected grains) varieties, while the most contaminated grains were presented by the Combin (on disinfected grains) and Levis (on non-disinfected grains) varieties. It is recognized that resistance against Fusarium head blight is influenced by physiological resistance factors and passive factors (e.g., morphological or developmental traits), which influence infection and disease development. Moreover, genes controlling resistance to FHB and those that affect plant height are associated. Gene coding for a shorter stem promoting sensitivity to FHB probably is due to pleiotropy. The presence of semi-dwarfing genes $R h t-B 1$ and $R h t-D 1$ influence the sensitivity to FHB [18]. It is conceivable that all these resistance factors also influence grain contamination.

Disinfection of grains is thought to reduce the number of fungi residing on the surface of the grain, thus allowing detecting fungi residing in the lower layers of the teguments. Little is known about the physiological activities and the ecological niche behaviour of fungi residing on grains [9]. Growth and colonization of the rest of the grain is certainly dependent on the ripening state of the grain and the availability of water $[19,20]$. The present results indicate that the fungal habitat on the surface or inside the grain is independent from wheat variety.

\section{Conclusions}

1. Colonization, abundance, and diversity of fungi on wheat grains are influenced by varietal factors other than Fusarium head blight resistance.

2. Future studies on grain contamination must consider the factor variety. This is particularly important when studying mycotoxin-producing species.

3. The specific morphological and physiological factors that govern the fungal colonization of grain should be further studied and considered in future risk-assessment studies. If future studies reveal the necessity, this information may be integrated in wheat-breeding programs to enhance consumer safety.

\section{Acknowledgements}

We gratefully acknowledge the Department of Plant Protection, Division of Plant Pathology and Mycology of Wrocław University of Environmental and Life Sciences for their support, including the use of its infrastructure. Many thanks to Stefan Kellenberger and Pierre Pignon from Agroscope in Changins for planting, harvesting, and conditioning the grains. This work was initiated within two travel grants from the ENDURE Network of Excellence that facilitated the exchange of personnel and ideas between IHAR and Agroscope Changins-Wädenswil.

\section{References}

1. PUSZ W. Morpho-physiological and molecular analyses of Alternaria alternata isolated from seeds of Amaranthus. Phytopathologia 54, 5, 2009.

2. KUMAR V., BASU M.S., RAJENDRAN T.P. Mycotoxin research and mycoflora in some commercially important agricultural commodities. Crop Protection, 27, 891, 2008.

3. PAUL P.A., EL-ALLAF S.M., LIPPS P.E., MADDEN L.V. Rain splash dispersal of Gibberella zeae within wheat canopies in Ohio. Phytopathology, 94, 1342, 2004.

4. ELMHOLT S. Ecology of the Ochratoxin A producing Penicillium verrucosum: Occurrence in field soil and grain with special attention to farming system and on-farm drying practices. Biological Agriculture and Horticulture, 20, 311, 2003.

5. CHAMPEIL A., FOURBET J.F., DORE T., ROSSIGNOL L. Influence of cropping system on Fusarium head blight and mycotoxin levels in winter wheat. Crop Protection, 23, 531, 2004.

6. ŪSELE G., BEINAROVIČA I., MEŽAKA I., LEGZDIN̦A L. Comparison of spring barley (Hordeum vulgare L.) screening methods for Fusarium head blight resistance breeding. Zemdirbyste-Agriculture, 100 (3), 31, 2013.

7. OSBORNE L.E., STEIN J.M. Epidemiology of Fusarium head blight on small-grain cereals. International Journal of Food Microbiology, 119 (1-2), 103, 2007.

8. ELSGAARD L., BØRGESEN C.D., JE J.E.O., SIEBERT S., EWERT F., PELTONEN-SAINIO P., RÖTTER R.P., SKJELVÅG A.O. Shifts in comparative advantages for maize, oat and wheat cropping under climate change in Europe. Addit Contam A: Chemistry, analysis, control, exposure \& risk assessment, 29, 1514, 2012.

9. XU X.M., NICHOLSON P. Community ecology of fungal pathogens causing wheat head blight. Annual Review of Phytopathology, 47, 83, 2009.

10. KANG Z., BUCHENAUER H., HUANG L., HAN Q., ZHANG CH. Cytological and immunocytochemical studies on responses of wheat spikes of the resistant Chinese cv. Sumai 3 and the susceptible cv. Xiaoyan 22 to infection by Fusarium graminearum. European Journal of Plant Pathology, 120 (4), 383, 2008.

11. HÄLLER GÄRTNER B., MUNICH M., KLEIJER G., MASCHER F. Characterisation of kernel resistance against Fusarium infection in spring wheat by baking quality and mycotoxin assessments. European Journal of Plant Pathology, 120, 61, 2008.

12. MASCHER F., MATASCI C., KNEUBUEHLER Y., KELLENBERGER S., QUIJANO C.D., KELLER B., SAUTTER C., SCHORI A. Resistance to fungal diseases of trans- 
genic wheat lines in the field (La résistance aux maladies fongiques de lignées de blé transgénique en plein champ). Recherche Agronomique Suisse, 3, 298, 2012 [In French].

13. SEMASKIENE R., MANKEVICIENE A., DABKEVICIUS Z., LEISTRUMAITE A. Toxic fungi infection and mycotoxin level in organic grain. Botanica Lithuanica, 7, 17, 2005.

14. LOGRIECO A., MORETTI A., SOLFRIZZO M. Alternaria toxins and plant diseases: an overview of origin, occurrence and risks. World Mycotox Journal, 2, 129, 2009.

15. BUERSTMAYR H., LEMMENS M., SCHMOLKE M., ZIMMERMANN G., HARTL L., MASCHER F., TROTTET M., GOSMAN N.E., NICHOLSON P. Multi-environment evaluation of level and stability of FHB resistance among parental lines and selected offspring derived from several European winter wheat mapping population. Plant Breeding, 127, 325, 2008.

16. SKINNES H., SEMAGN K., TARKEGNE Y., MARØY A.G., BØJRNSTAD A. 2010. The inheritance of anther extrusion in hexaploid wheat and its relationship to Fusarium head blight resistance and deoxynivalenol content. Plant Breeding, 129, 149, 2010.
17. KUBO K., FUJITA M., KAWADA N., NAKAJIMA T., NAKAMURA K., MAEJIMA H., USHIYAMA T., HATTA K., MATSUNAKA H. Minor differences in anther extrusion affect resistance to Fusarium head blight in wheat. Journal of Phytopathology, 161, 308, 2013.

18. SRINIVASACHARY N., GOSMAN A., STEED A., SIMMONDS J., LEVERINGTON-WAITE M., WANG Y., SNAPE J., NICHOLSON P. Susceptibility to Fusarium head blight is associated with the Rht-D1b semi-dwarfing allele in wheat. Theoretical and Applied Genetics, 116, 1145, 2008.

19. MAGAN N., ALDRED D., MYLONA K., LAMBERT R.J.W. Limiting mycotoxins in stored wheat - a review. Food additives and contaminants, 27, 644, 2010.

20. XU X.M., NICHOLSON P., THOMSETT M.A., SIMPSON D., COOKE B.M., DOOHAN F.M., BRENNAN J., MONAGHAN S., MORETTI A., MULE G., HORNOK L., BEKI E., TATNELL J., RITIENI A., EDWARDS S.G. Relationship between the fungal complex causing Fusarium head blight of wheat and environmental conditions. Phytopathology, 98, 69, 2008. 